\title{
Semi-Boolean Steiner quadruple systems and dimensional dual hyperovals
}

\author{
Marco Buratti and Alberto Del Fra \\ Dedicated to Professor Adriano Barlotit on the occasion of his 80 th birthday
}

\begin{abstract}
A dimensional dual hyperoval satisfying property $(H)[6]$ in a projective space of order 2 is naturally associated with a "semi-Boolean" Steiner quadruple system. The only known examples are associated with Boolean systems. For every $d>2$, we construct a new $d$ dimensional dual hyperoval satisfying property $(\mathrm{H})$ in $\mathrm{PG}(d(d+3) / 2,2)$; its related semiBoolean system is the Teirlinck one. It is universal and admits quotients in $\operatorname{PG}(n, 2)$, with $4 d<n<d(d+3) / 2$, if $d \geqslant 6$. We also prove the uniqueness of $d$-dimensional dual hyperovals satisfying property $(\mathrm{H})$ in $\mathrm{PG}(d(d+3) / 2,2)$, whose related semi-Boolean systems belongs to a particular class, which includes Boolean and Teirlinck systems. Finally, we prove property (mI) [6] for them.
\end{abstract}

\section{Introduction}

1.1 Definitions and preliminary results. As in [7], a d-dimensional dual hyperoval is a family $F$ of $d$-dimensional subspaces in a projective space $\Pi$ of dimension $n$ and order $q$ satisfying the following conditions:

(i) every point of $\Pi$ belongs to either 0 or 2 members of $F$;

(ii) any two members of $F$ have precisely a point in common;

(iii) the set of points belonging to the members of $F$ spans $\Pi$.

Since the number $\theta(d, q)$ of points of $\operatorname{PG}(d, q)$ is equal to $\left(q^{d+1}-1\right) /(q-1)$, it follows by (i) and (ii) that $|F|=\theta(d, q)+1=\left(q^{d+1}+q-2\right) /(q-1)$.

When $n=2$ and $d=1$, we get the classical definition of dual hyperoval in a projective plane. In that case $q$ is necessarily even. This property has been generalized in [4] for any $d$-dimensional dual hyperoval in $\operatorname{PG}(n, q)$ with $n$ odd.

$d$-dimensional dual hyperovals appear as a particular case of $d$-dimensional dual $\operatorname{arcs}$, defined in [11] substituting (i) with

(j) every point of $\Pi$ belongs to at most 2 members of $F$. 
For any result on $d$-dimensional dual arcs, we refer to [12]. In this paper we restrict the subject to $d$-dimensional dual hyperovals. We also assume $d>1$, since $d=1$ corresponds to the usual dual hyperovals in projective planes. We use the terminology dimensional dual hyperoval if we do not need to point out the dimension $d$.

Yoshiara proved in [12] that $D=d(d+3) / 2$ and $D+2$ are upper bounds for the dimension of a projective space of order $q$, containing a $d$-dimensional dual hyperoval, in the cases $q>2$ and $q=2$, respectively.

For $q=2^{h}, h>1$, this is the best possible, since Yoshiara also constructed in [12] a $d$-dimensional dual hyperoval, for any $d$, by completing a $d$-dimensional dual arc defined by Thas and Van Maldeghem in [9].

The upper bound $D+2$ in the case $q=2$ maybe could be improved. In fact the $d$-dimensional dual hyperovals, which are known until now in projective spaces of order 2, also suggest $D$ as upper bound.

As in [6], we give the following definition.

Definition 1.1. Given a $d$-dimensional dual hyperoval $F$ and an $m$-subspace $W$ in $\operatorname{PG}(n, q)$, if the family $F^{\prime}$ obtained factorizing $F$ by $W$ is a $d$-dimensional dual hyperoval in $\mathrm{PG}(n-m-1, q)$, we say that $F^{\prime}$ is a quotient of $F$. A dimensional dual hyperoval which is not a quotient of any other one is called universal. A dimensional dual hyperoval is minimal if does not admit any quotient.

We get the following result for the existence of quotients of dimensional dual hyperovals in projective spaces of order 2 .

Theorem 1.2. $A$ d-dimensional dual hyperoval $F$ in $\mathrm{PG}(n, 2)$, with $n>4 d$, admits a quotient in $\mathrm{PG}(k, 2), 4 d \leqslant k \leqslant n$.

Proof. We recall that a $d$-dimensional dual hyperoval $F$ in $\mathrm{PG}(n, 2)$ can be factorized in a $d$-dimensional dual hyperoval in $\mathrm{PG}(n-i-1,2)$ if and only if there is an $i$ subspace of $\operatorname{PG}(n, 2)$ disjoint with the union, say $L$, of the $2 d$-spaces spanned by the pairs of distinct $d$-spaces of $F([6],[12])$.

Since the number of pairs of $d$-spaces of $F$ is $2^{d}\left(2^{d+1}-1\right)$ and the number of points of a $2 d$-space is $2^{2 d+1}-1$, we have:

$$
|L|<2^{d}\left(2^{d+1}-1\right)\left(2^{2 d+1}-1\right)<2^{4 d+2}-1=|\mathrm{PG}(4 d+1,2)|
$$

Since $n \geqslant 4 d+1$, the complement $\mathscr{C} L$ of $L$ in $\mathrm{PG}(n, 2)$ is not empty. Therefore, factorizing by a point of $\mathscr{C} L$, we get a $d$-dimensional dual hyperoval of $\operatorname{PG}(n-1,2)$. By induction we get the statement.

Definition 1.3. A dimensional dual hyperoval $F$ in $\mathrm{PG}(n, q)$ is said to satisfy property $(\mathrm{H})$ if, for any triple of distinct members of $F$, denoting by $\pi$ the plane containing their pairwise intersection points, the set of lines of $\pi$ contained in some member of $F$ is a dual hyperoval (then $q$ is even). 
Note that any quotient of a dimensional dual hyperoval defined as above also satisfies property $(\mathrm{H})$.

Definition 1.4. A $d$-dimensional dual hyperoval $F$ is said to satisfy property $(\mathrm{mI})$ if, for any triple of distinct members of $F$, the intersection between the $2 d$-space spanned by two of them and the third one is a line.

Property $(\mathrm{mI})$ is not necessarily preserved by quotients. Property $(\mathrm{mI})$ is denoted by $(\mathrm{T})$ in [4] and in [12]. Huybrechts proved in [6] that $(\mathrm{mI})$ implies $(\mathrm{H})$.

It is immediate to see that property $(\mathrm{H})$ allows to group the members of $F$ in blocks of size $q+2$ such that any $q+1$ members of a block $B$ meet the remaining member of $B$ in $q+1$ points belonging to the same line. These blocks give rise to a $\left(3, q+2,\left(q^{d+1}+q-2\right) /(q-1)\right)$ Steiner system (whose points are the members of $F$ ) whose derived systems are isomorphic to $\mathrm{PG}_{1}(d, q)$, the point-line system associated with $\operatorname{PG}(d, q)$. We recall that, enriching such a Steiner system by all possible pairs of distinct points, called lines, we get a geometry belonging to the diagram c. $\mathrm{PG}_{1}(d, q)$.

We remark that any quotient of a dimensional dual hyperoval $F$ satisfying property $(\mathrm{H})$ gives rise to the same Steiner system related to $F$.

For $q=2$ a Steiner system as above is said to be a semi-Boolean Steiner quadruple system of order $2^{d+1}\left(\mathrm{SBQS}\left(2^{d+1}\right)\right.$ in short). A particular case of $\mathrm{SBQS}\left(2^{d+1}\right)$ is the Boolean Steiner quadruple system of order $2^{d+1}$, i.e., the point-plane system associated with the affine space of dimension $d+1$ and order 2 .

In [1] an explicit construction of SBQSs is proposed. Here we describe a particular case of it. Let $V$ be the $(d+1)$-dimensional vector space over $\mathbb{Z}_{2}$ and let $z$ be an element chosen in $V-\{0\}$. Given $X \subseteq V-\{0, z\}$ such that $x \in X$ if and only if $x+z \in X$, consider the map $q$ from $\left(\begin{array}{c}V \\ 3\end{array}\right)$ to $V$ defined as follows:

$$
q(\{a, b, c\})=a+b+c+[\chi(a+b)+\chi(a+c)+\chi(b+c)] z,
$$

where $\chi$ is the characteristic function of $X$. Set $\mathscr{Q}(X)=\{\{a, b, c, q(\{a, b, c\})\} \mid$ $\left.\{a, b, c\} \in\left(\begin{array}{l}V \\ 3\end{array}\right)\right\}$. In [1] it is proved that the pair $(V, \mathcal{Q}(X))$ is an SBQS. In the following such a system will be called characteristic. Since in [1] it is also proved that there are at least $2^{\lfloor 3(d-3) / 2\rfloor}$ characteristic $\operatorname{SBQS}\left(2^{d+1}\right)$, we may state the following theorem.

Theorem 1.5. For any $d \geqslant 3$, there exist at least $2^{\lfloor 3(d-3) / 2\rfloor}$ geometries belonging to the diagram c. $\mathrm{PG}_{1}(d, 2)$.

Remark 1.6. The Boolean $\mathrm{SBQS}\left(2^{d+1}\right)$ is characteristic, since it corresponds to the case $X=\varnothing$. Also, the $\operatorname{SBQS}\left(2^{d+1}\right)$ as defined by Teirlinck in [8] is characteristic since it may be viewed as $(V, \mathscr{Q}(X))$ with $X=V-\{0, z\}$.

1.2 Examples of dimensional dual hyperovals. We give a list of the dimensional dual hyperovals which are known until now. 


\begin{tabular}{c|c|c|c|c|c} 
& author & $d$ & $n$ & $q$ & reference \\
\hline $\mathrm{a}$ & Yoshiara & any & $d(d+3) / 2$ & $2^{h}$ & {$[12]$} \\
\hline $\mathrm{b}$ & Huybrechts & any & $d(d+3) / 2$ & 2 & {$[6]$} \\
\hline $\mathrm{c}$ & Yoshiara & any & $2 d$ and $2 d+1$ & 2 & {$[10]$} \\
\hline $\mathrm{d}$ & Cooperstein-Thas & any & $2 d$ & 2 & {$[3]$} \\
\hline $\mathrm{e}$ & Huybrechts-Pasini & 2 & 5 & 4 & {$[7]$ and [2] } \\
\hline $\mathrm{f}$ & Buratti-Del Fra & any & $d(d+3) / 2$ & 2 & this paper
\end{tabular}

Property $(\mathrm{mI})$ (and consequently property $(\mathrm{H})$ ) always holds in cases $\mathrm{b}$ ), e) and $\mathrm{f}$ ), while in case c) it holds only if $(d, n)=(2,5)$. Property $(\mathrm{H})$ also holds, for each $d$, for exactly one $d$-dimensional dual hyperoval of c) in dimension $2 d+1$. All these dimensional dual hyperovals satisfying $(\mathrm{H})$ are related to the Boolean Steiner system. Property $(\mathrm{H})$ (and consequently property $(\mathrm{mI})$ ) does not hold for any dimensional dual hyperoval of a), as proved by Yoshiara (private communication).

The $d$-dimensional dual hyperovals of b) admit quotients in $\operatorname{PG}(n, 2)$, for every $n$ with $2 d+1 \leqslant n \leqslant D=d(d+3) / 2$ ([12], Theorem 14). Those of $\mathrm{f}$ ) admit quotients in $\mathrm{PG}(n, 2)$, for every $n$ with $4 d \leqslant n \leqslant D$, if $d \geqslant 6$ (Theorem 3.4). The $d$-dimensional dual hyperovals of a) also admit quotients in $\operatorname{PG}\left(n, 2^{h}\right)$, for every $n$ with $4 d-2 \leqslant$ $n \leqslant D$ ([12], Proposition 15).

We refer the reader to the papers recalled in the above table for more information about the examples. Here, we describe explicitly only the dimensional dual hyperovals of b). Consider in $\operatorname{PG}(D, 2)$ the Grassmann variety of lines of $\operatorname{PG}(d+1,2)$ $(D=d(d+3) / 2)$. The image of the point set of a copy of $\mathrm{AG}(d+1,2)$ inside $\mathrm{PG}(d+1,2)$, under the Grassmann transformation, is a $d$-dimensional dual hyperoval of $\mathrm{PG}(D, 2)$.

1.3 Dimensional dual hyperovals and c.AG* geometries. As remarked in [7], every $d$-dimensional dual hyperoval $F$ gives rise to a geometry belonging to the diagram c. $\mathrm{AG}_{d+1}^{*}$, where $\mathrm{AG}_{d+1}^{*}$ represents the dual of the point-line system of a $(d+1)$ dimensional affine space. This geometry satisfies property (LL), and also satisfies property (T) (see [7], §1.5) if and only if $F$ satisfies property (mI). If $q=2$, the previous diagram becomes c.c* and the corresponding geometries are semibiplanes.

\section{Characteristic dimensional dual hyperovals}

As in the previous section let $V=\mathbb{Z}_{2}^{d+1}$ and let $X$ be a subset of $V-\{0, z\}$ ( $z$ a nonzero element of $V$ ), with the property that $x \in X$ implies that $x+z \in X$.

Let $\mathscr{B}$ be a basis of $V$ containing $z$ and set $\mathscr{G}=\mathscr{B} \cup\{0\}$. Every vector of $V$ is the sum of an odd number of elements of $\mathscr{G}$, in a unique way. We denote by $J(v)$ the odd subset of $\mathscr{G}$ related to the vector $v$.

Recall that the symmetric difference $A \triangle B$ between two sets $A, B$ is defined by 
$A \triangle B=(A \cup B)-(A \cap B)$. More generally, given $A_{1}, \ldots, A_{n}$, the symmetric difference $A_{1} \triangle \cdots \triangle A_{n}$ is the set of elements belonging to an odd number of $A_{i}$ 's.

For every ordered pair $(u, v)$ of elements of $V$, let us define:

$$
x_{u, v}=\left[\mid X \cap\left(\{u+w\}_{w \in J(v)} \Delta\{u+v\}\right) \|,\right.
$$

where $[y]$ means the residue class of $y$ modulo 2. Note that:

$$
\{v, v+z\} \cap \mathscr{G} \neq \varnothing \Rightarrow x_{u, v}=0 .
$$

We remark that, for any $u, v \in V$

$$
x_{u, v}=x_{u, v+z}=x_{u+z, v}=x_{u+z, v+z}
$$

and, in particular,

$$
x_{u, 0}=x_{u, z}, \quad x_{0, u}=x_{z, u} .
$$

We point out that, in the following, all equalities involving $x_{u, v}$ 's are understood in $\mathbb{Z}_{2}$. By abuse of notation, we will write $y$ instead of the residue class $[y]$.

Proposition 2.1. For every triple of elements $u, v_{1}, v_{2}$ in $V$, we have

$$
x_{u, v_{1}}+x_{u, v_{2}}+x_{u, u}+x_{u, u+v_{1}+v_{2}}=\left|X \cap\left\{u+v_{1}, u+v_{2}, v_{1}+v_{2}\right\}\right| .
$$

Proof. Using (2) we get

$$
x_{u, v_{1}}+x_{u, v_{2}}+x_{u, u}=\sum_{i=1}^{2}\left|X \cap\left(\{u+w\}_{w \in J\left(v_{i}\right)} \Delta\left\{u+v_{i}\right\}\right)\right|+\left|X \cap\{u+w\}_{w \in J(u)}\right| .
$$

Since $J\left(v_{1}+v_{2}+u\right)=J\left(v_{1}\right) \triangle J\left(v_{2}\right) \triangle J(u)$, we also have

$$
x_{u, u+v_{1}+v_{2}}=\left|X \cap\left(\{u+w\}_{w \in J\left(v_{1}\right)} \Delta\{u+w\}_{w \in J\left(v_{2}\right)} \Delta\{u+w\}_{w \in J J(u)} \Delta\left\{v_{1}+v_{2}\right\}\right)\right| .
$$

From (7), (8) we deduce the statement.

Let $F$ be a $d$-dimensional dual hyperoval in $\operatorname{PG}(n, 2)$ satisfying property $(\mathrm{H})$. Assume that its associated SBQS, say $Q$, is characteristic and related to $X \subseteq V-$ $\{0, z\}$ ( $z$ a non-zero element of $V$ such that $x \in X \Rightarrow x+z \in X)$. Such a structure will be called a characteristic d-dimensional dual hyperoval.

For every $v \in V$, let $S_{v}$ be the $d$-space of $F$ associated with $v$. Given two $d$-spaces $S_{u}, S_{v}$ in $F$, denote by $a_{u, v}$ (or indifferently by $a_{v, u}$ ) the intersection point $S_{u} \cap S_{v}$. 
From now on we will work in $\mathbb{Z}_{2}^{n+1}$ that, by abuse of notation, will be identified with $\operatorname{PG}(n, 2) \cup\{0\}$. For every $u \in V$, we set:

$$
a_{u, u}=0 .
$$

Given pairwise distinct $u, v_{1}, v_{2}$ in $V$, in view of property $(\mathrm{H})$ we have

$$
a_{u, v_{1}}+a_{u, v_{2}}=a_{u, q\left(\left\{u, v_{1}, v_{2}\right\}\right)}
$$

where $q\left(\left\{u, v_{1}, v_{2}\right\}\right)$ is the 4 th point of the block of $Q$ determined by $u, v_{1}, v_{2}$. Since $Q$ is characteristic, equalities (10), (9) and (1) imply

$$
a_{u, v_{1}}+a_{u, v_{2}}= \begin{cases}a_{u, u+v_{1}+v_{2}} & \text { if }\left|X \cap\left(\left\{u+v_{1}\right\} \Delta\left\{u+v_{2}\right\} \triangle\left\{v_{1}+v_{2}\right\}\right)\right| \\ & \text { is even, } \\ a_{u, u+v_{1}+v_{2}+z} & \text { otherwise. }\end{cases}
$$

Thus, chosen $v_{1}, v_{2}, \ldots, v_{i}, i>2$,

-if $i$ is even, we have

$$
\begin{aligned}
& a_{u, v_{1}}+a_{u, v_{2}}+\cdots+a_{u, v_{i}} \\
& = \begin{cases}a_{u, u+v_{1}+\cdots+v_{i}} & \text { if }\left|X \cap\left(\left\{u+v_{1}\right\} \Delta \cdots \Delta\left\{u+v_{i}\right\} \triangle\left\{v_{1}+\cdots+v_{i}\right\}\right)\right| \\
a_{u, u+v_{1}+\cdots+v_{i}+z} & \text { is even, }\end{cases}
\end{aligned}
$$

-if $i$ is odd, we have

$$
\begin{aligned}
a_{u, v_{1}} & +a_{u, v_{2}}+\cdots+a_{u, v_{i}} \\
& = \begin{cases}a_{u, v_{1}+\cdots+v_{i}} & \text { if }\left|X \cap\left(\left\{u+v_{1}\right\} \Delta \cdots \Delta\left\{u+v_{i}\right\} \triangle\left\{u+v_{1}+\cdots+v_{i}\right\}\right)\right| \\
a_{u, v_{1}+\cdots+v_{i}+z} & \text { is even, }\end{cases}
\end{aligned}
$$

We remark that, for any $u, v$ in $V$, we get by (13)

$$
a_{u, v+z}=a_{u, v}+a_{u, 0}+a_{u, z}
$$

For any ordered pair $(u, v)$ of elements of $V$, we get by (2), (13) and (14)

$$
a_{u, v}=\sum_{w \in J(v)} a_{u, w}+x_{u, v}\left(a_{u, 0}+a_{u, z}\right)
$$

By iterated use of (15) and (4), we get 


$$
\begin{aligned}
a_{u, v}= & \sum_{w \in J(v)} a_{w, u}+x_{u, v}\left(a_{0, u}+a_{z, u}\right) \\
= & \sum_{w \in J(v), w^{\prime} \in J(u)} a_{w, w^{\prime}}+\sum_{w \in J(v)} x_{w, u}\left(a_{w, 0}+a_{w, z}\right) \\
& +x_{u, v}\left[\sum_{w \in J(u)} a_{0, w}+x_{0, u}\left(a_{0,0}+a_{0, z}\right)+\sum_{w \in J(u)} a_{z, w}+x_{z, u}\left(a_{z, 0}+a_{z, z}\right)\right] \\
= & \sum_{w \in J(v), w^{\prime} \in J(u)} a_{w, w^{\prime}}+\sum_{w \in J(v)} x_{w, u}\left(a_{w, 0}+a_{w, z}\right)+x_{u, v} \sum_{w \in J(u)}\left(a_{0, w}+a_{z, w}\right) .
\end{aligned}
$$

In conclusion, for any $u, v \in V$ we have:

$$
a_{u, v}=\sum_{\substack{w \in J(v) \\ w^{\prime} \in J(u)}} a_{w, w^{\prime}}+\sum_{w \in J(v)} x_{w, u}\left(a_{w, 0}+a_{w, z}\right)+x_{u, v} \sum_{w \in J(u)}\left(a_{0, w}+a_{z, w}\right) .
$$

Note that from (14) we also deduce

$$
a_{u, v+z}=a_{u, v}+\sum_{w \in J(u)}\left(a_{w, 0}+a_{w, z}\right)
$$

and in particular

$$
a_{u, u+z}=\sum_{w \in J(u)}\left(a_{w, 0}+a_{w, z}\right)
$$

The previous results allow to get the following upper bound for the dimension of a projective space containing a characteristic $d$-dimensional dual hyperoval:

Theorem 2.2. If a characteristic d-dimensional dual hyperoval $F$ exists in $\mathrm{PG}(n, 2)$, then $n \leqslant d(d+3) / 2$.

Proof. In view of (iii) in Section 1, it is enough to prove that the set $\left\{a_{w, w^{\prime}} \mid w, w^{\prime} \in\right.$ $\left.\mathscr{G}, w \neq w^{\prime}\right\}$ of size $\left(\begin{array}{c}d+2 \\ 2\end{array}\right)$ spans all the points $a_{u, v}$ of the $d$-spaces of $F$. The statement follows from equality (16).

Remark 2.3. Equality (16) shows that a characteristic dimensional dual hyperoval in $\operatorname{PG}(d(d+3) / 2,2)$ is completely determined by the characteristic SBQS related to it. In particular, for any $d$, the only $d$-dimensional dual hyperoval in $\mathrm{PG}(d(d+3), 2)$ whose related SBQS is Boolean belongs to the Huybrechts' family.

Remark 2.4. For any $u, v \in V$, equalities $a_{u, u}=0$ and $a_{u, v}=a_{v, u}$ imply, by (16), strong conditions on the values $x_{u, v}$ and consequently on the SBQS associated with a char- 
acteristic dimensional dual hyperoval. We already know that the Boolean system of order $2^{d+1}$ is suitable for a $d$-dimensional dual hyperoval (the Huybrechts' one). Now, we prove that the Teirlinck $\operatorname{SBQS}\left(2^{d+1}\right)$ is also related to a $d$-dimensional dual hyperoval.

Theorem 2.5. Let $Q$ be the Boolean or the Teirlinck system $S\left(3,4,2^{d+1}\right)$. Up to isomorphism, there is exactly one d-dimensional dual hyperoval in $\mathrm{PG}(d(d+3) / 2,2)$, satisfying property $(\mathrm{H})$ and admitting $Q$ as related $S B Q S$.

Proof. The uniqueness has been noticed in Remark 2.3. The existence in the Boolean case is clear in view of the Huybrechts' family. It remains to prove the existence in the

case when $Q$ is a Teirlinck system.
$\quad$ Take an arbitrary bijection $\alpha$ between $\left(\begin{array}{c}\mathscr{G} \\ 2\end{array}\right)$ and a fixed basis of $W=\mathbb{Z}_{2}^{\left(\begin{array}{c}d+2 \\ 2\end{array}\right)}$ and set:

$$
b_{w, w^{\prime}}=b_{w^{\prime}, w}=\alpha\left(\left\{w, w^{\prime}\right\}\right) \quad \text { for all }\left\{w, w^{\prime}\right\} \in\left(\begin{array}{l}
\mathscr{G} \\
2
\end{array}\right) .
$$

We also set $b_{w, w}=0$ for every $w \in \mathscr{G}$.

For every pair $\{u, v\}$ of elements of $V$, we define $b_{u, v}$ as suggested by (16):

$$
b_{u, v}=\sum_{\substack{w \in J(u) \\ w^{\prime} \in J(v)}} b_{w, w^{\prime}}+\sum_{w \in J(v)} x_{w, u}\left(b_{w, 0}+b_{w, z}\right)+x_{u, v} \sum_{w \in J(u)}\left(b_{w, 0}+b_{w, z}\right)
$$

We note that (20) is an identity for $u, v$ in $\mathscr{G}$, since in this case $J(u)=\{u\}, J(v)=\{v\}$ and $x_{u, v}=x_{v, u}=0$ by (3).

We recall that the Teirlinck system is related to the set $X=V-\{0, z\}$ (see Remark 1.6). Since the size of $\left\{\{u+w\}_{w \in J(v)} \triangle\{u+v\}\right\}$ is even, by (2) we have $x_{u, v}=1$ if and only if $\{v, v+z\} \cap \mathscr{G}=\varnothing$ and one of the following conditions holds:
A) $\{0, z\} \nsubseteq J(v)$ and $\{u, u+z\} \cap J(v) \neq \varnothing$;
B) $\{0, z\} \subseteq J(v)$ and $\{u, u+z\} \cap(J(v)-\{0, z\}) \neq \varnothing$;
C) $u \in\{v, v+z\}$.

Hence, a straightforward computation shows that (20) implies

$$
\begin{aligned}
b_{u, u} & =0 & & \text { for all } u \in V, \\
b_{u, u+z} & =\sum_{w \in J(u)}\left(b_{w, 0}+b_{w, z}\right) & & \text { for all } u \in V
\end{aligned}
$$

and for any $u, v$ with $u \notin\{v, v+z\}$

$$
b_{u, v}=\sum_{\substack{w \in J(u) \\ w^{\prime} \in J(v)}} b_{w, w^{\prime}}+\sum_{w \in(J(u) \cap J(v))-\{0, z\}}\left(b_{w, 0}+b_{w, z}\right)+i_{u, v} b_{0, z}
$$


with $i_{u, v} \in \mathbb{Z}_{2}$ defined by

$$
i_{u, v}=0 \Leftrightarrow\{0, z\} \subset J(y) \text { for some } y \in\{u, u+z, v, v+z\} .
$$

It follows that, for any $u, v$ in $V$

$$
b_{u, v}=b_{v, u} .
$$

If $b_{u, v} \neq 0,(23)$ allows to express $b_{u, v}$ as a sum of some pairwise distinct elements $b_{p, q}$, with $p, q \in \mathscr{G}$. This sum will be called reduced sum of $b_{u, v}$. If $b_{u, v}=0$, we will say that the reduced sum of $b_{u, v}$ is null.

Let us prove that $u \neq v$ implies $b_{u, v} \neq 0$. Let $v \neq u+z$. If $v=0$, equality (23) becomes $b_{u, 0}=\sum_{w \in J(u)} b_{w, 0}+i_{u, 0} b_{0, z} \neq 0$ since $J(u)-\{z\} \neq \varnothing$. We get the analogous result for $v=z$ or $u=0$ or $u=z$. If both $u$ and $v$ are different from 0 and $z$, by (23) there is at least one $b_{w, w^{\prime}}$ with $w, w^{\prime} \in \mathscr{G}-\{0, z\}$ appearing in the reduced sum of $b_{u, v}$. On the other hand (22) implies $b_{u, u+z} \neq 0$. Thus, in every case, from $u \neq v$ we deduce $b_{u, v} \neq 0$.

For any $u, v$ in $V$, a straightforward but quite tedious computation shows that (21), (22) and (23) imply

$$
b_{u, v}=\sum_{w \in J(v)} b_{u, w}+x_{u, v}\left(b_{u, 0}+b_{u, z}\right)
$$

Given three pairwise distinct elements $u, v_{1}, v_{2}$ in $V$, from (26) and (21) it follows:

$$
\begin{gathered}
b_{u, v_{i}}=\sum_{w \in J\left(v_{i}\right)} b_{u, w}+x_{u, v_{i}}\left(b_{u, 0}+b_{u, z}\right) \quad(i=1,2), \\
\sum_{w \in J(u)} b_{u, w}=x_{u, u}\left(b_{u, 0}+b_{u, z}\right), \\
b_{u, u+v_{1}+v_{2}}=\sum_{w \in J\left(u+v_{1}+v_{2}\right)} b_{u, w}+x_{u, u+v_{1}+v_{2}}\left(b_{u, 0}+b_{u, z}\right) .
\end{gathered}
$$

Recalling that $J\left(u+v_{1}+v_{2}\right)=J(u) \triangle J\left(v_{1}\right) \triangle J\left(v_{2}\right)$, we deduce from (27), (28) and (29)

$$
\begin{aligned}
b_{u, u+v_{1}+v_{2}} & =\sum_{w \in J\left(v_{1}\right)} b_{u, w}+\sum_{w \in J\left(v_{2}\right)} b_{u, w}+\sum_{w \in J(u)} b_{u, w}+x_{u, u+v_{1}+v_{2}}\left(b_{u, 0}+b_{u, z}\right) \\
& =b_{u, v_{1}}+b_{u, v_{2}}+\sum_{w \in J(u)} b_{u, w}+\left(x_{u, v_{1}}+x_{u, v_{2}}+x_{u, u+v_{1}+v_{2}}\right)\left(b_{u, 0}+b_{u, z}\right) \\
& =b_{u, v_{1}}+b_{u, v_{2}}+\left(x_{u, v_{1}}+x_{u, v_{2}}+x_{u, u}+x_{u, u+v_{1}+v_{2}}\right)\left(b_{u, 0}+b_{u, z}\right),
\end{aligned}
$$

whence 


$$
b_{u, v_{1}}+b_{u, v_{2}}=b_{u, u+v_{1}+v_{2}}+\left(x_{u, v_{1}}+x_{u, v_{2}}+x_{u, u}+x_{u, u+v_{1}+v_{2}}\right)\left(b_{u, 0}+b_{u, z}\right) .
$$

Since $b_{u, u+v_{1}+v_{2}+z}=b_{u, u+v_{1}+v_{2}}+b_{u, 0}+b_{u, z}$, we get from (6) and (30)

$$
b_{u, v_{1}}+b_{u, v_{2}}= \begin{cases}b_{u, u+v_{1}+v_{2}} & \text { if }\left|X \cap\left\{u+v_{1}, u+v_{2}, v_{1}+v_{2}\right\}\right| \text { is even } \\ b_{u, u+v_{1}+v_{2}+z} & \text { otherwise }\end{cases}
$$

that is by (1)

$$
b_{u, v_{1}}+b_{u, v_{2}}=b_{u, q\left(\left\{u, v_{1}, v_{2}\right\}\right)} .
$$

For every $u \in V$, define $S_{u}=\left\{b_{u, v}: v \in V\right\}$. By (32) $S_{u}$ is a subspace of $W$, which, by (26), is spanned by the elements $b_{u, w}$ with $w \in \mathscr{G}$.

For any $u \in V$ and $\bar{w} \in J(u)$, (28) implies

$$
b_{u, \bar{w}}=\sum_{w \in J(u)-\{\bar{w}\}} b_{u, w}+x_{u, u}\left(b_{u, 0}+b_{u, z}\right)
$$

Thus we deduce that the dimension (as vector space) of $S_{u}$ is at most $d+1$. Now, our goal is to prove that, for any choice of two distinct pairs of distinct elements of $V,\{u, v\},\left\{u^{\prime}, v^{\prime}\right\}$, we have $b_{u, v} \neq b_{u^{\prime}, v^{\prime}}$.

Partition the set of pairs $\{u, v\}, u \neq v$, in equivalence classes of type $\{\{u, v\}$, $\{u+z, v\},\{u, v+z\},\{u+z, v+z\}\}$. We denote such a class by $[\{u, v\}]$.

Note that (23) implies that any $w \in(J(u) \cup J(v))-\{0, z\}$ appears an even or odd number of times as index of some elements in the reduced sum of $b_{u, v}$, according to whether $w$ belongs to $J(u) \cap J(v)$ or $J(u) \triangle J(v)$, respectively.

This remark allows, starting from the reduced sum of $b_{u, v}$, to completely determine $J(u)-\{0, z\}, J(v)-\{0, z\}$, and therefore the equivalence class $[\{u, v\}]$. It follows that, if $\{u, v\},\left\{u^{\prime}, v^{\prime}\right\}$ do not belong to the same class, then $b_{u, v}$ and $b_{u^{\prime}, v^{\prime}}$ have distinct reduced sums, namely they are distinct.

Now we choose an equivalence class $[\{u, v\}]$. If $v=u+z$, this class is a singleton. If $v \neq u+z$, it is formed by four distinct pairs. In this case, from (23) we deduce

$$
b_{u, v+z}=b_{u, v}+b_{u, 0}+b_{u, z}=b_{u, v}+\sum_{w \in J(u)}\left(b_{w, 0}+b_{w, z}\right)
$$

whence

$$
\begin{gathered}
b_{u+z, v}=b_{u, v}+\sum_{w \in J(v)}\left(b_{w, 0}+b_{w, z}\right) \\
b_{u+z, v+z}=b_{u, v}+\sum_{w \in J(u)}\left(b_{w, 0}+b_{w, z}\right)+\sum_{w \in J(v)}\left(b_{w, 0}+b_{w, z}\right) .
\end{gathered}
$$

Then $b_{u, v}, b_{u, v+z}, b_{u+z, v}, b_{u+z, v+z}$ have pairwise distinct reduced sums. 


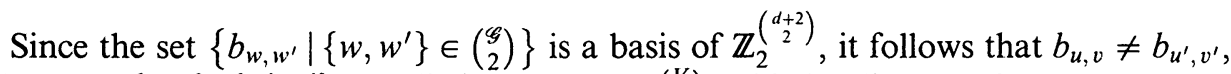
whenever $\{u, v\},\left\{u^{\prime}, v^{\prime}\right\}$ are distinct pairs of $\left(\begin{array}{l}V \\ 2\end{array}\right)$. This implies that for any two distinct $S_{u}, S_{v}$ we have $S_{u} \cap S_{v}=\left\{0, b_{u, v}\right\}$.

Now note that $\left|S_{u}\right|=\left|\left\{b_{u, v} \mid v \in V\right\}\right|=2^{d+1}$, so that $S_{u}$ has dimension $d+1$. Interpreting the previous results in $\mathrm{PG}(d(d+3) / 2,2)$, we deduce that the projective $d$ spaces corresponding to the $S_{u}$ 's form a $d$-dimensional dual hyperoval. It satisfies property (H) by (32).

\section{Universality and quotients}

Theorem 2.2 immediately implies:

Theorem 3.1. All characteristic d-dimensional dual hyperovals in $\mathrm{PG}(d(d+3) / 2,2)$ are universal.

Now, we prove that any characteristic $d$-dimensional dual hyperoval is quotient of a suitable one in $\operatorname{PG}(d(d+3) / 2,2)$.

Theorem 3.2. Any characteristic d-dimensional dual hyperoval $F$ in $\mathrm{PG}(n, 2), n<$ $d(d+3) / 2$, is the quotient of a characteristic d-dimensional dual hyperoval in $\mathrm{PG}(d(d+3) / 2,2)$, related to the same characteristic $S B Q S$.

Proof. We use the same notation as in the previous section. Since $n<d(d+3) / 2$, the set $A=\left\{a_{x, y}:\{x, y\} \in\left(\begin{array}{c}\mathscr{G} \\ 2\end{array}\right)\right\}$ is a set of generators but not a basis of $\operatorname{PG}(n, 2)$. Let $A^{\prime}$ be a basis contained in $A$, and take $a_{\bar{x}, \bar{y}}$ in $A-A^{\prime}$. Consider PG $(n, 2)$ as a hyperplane $\alpha$ in $\mathrm{PG}(n+1,2)$ and fix a point $p$ in $\mathrm{PG}(n+1,2)-\alpha$. Set

$$
\begin{aligned}
& a_{x, y}^{\prime}=a_{x, y} \quad \text { for } x, y \in \mathscr{G} \text { with }\{x, y\} \neq\{\bar{x}, \bar{y}\} \text { and } \\
& a_{\bar{x}, \bar{y}}^{\prime}=a_{\bar{x}, \bar{y}}+p .
\end{aligned}
$$

For any $a_{u, v}$ in $\operatorname{PG}(n, 2)$ consider equality (16) and define $a_{u, v}^{\prime}$ in $\operatorname{PG}(n+1,2)$ by replacing every $a_{x, y}(x, y \in \mathscr{G})$ with $a_{x, y}^{\prime}$ in the right hand side of (16).

For any fixed $u$, the elements $a_{u, v}^{\prime}$ again determine a $d$-space $S_{u}^{\prime}$, whose projection from $p$ on $\alpha$ is $S_{u}$. The family $F^{\prime}=\left\{S_{u}^{\prime}\right\}_{u \in V}$ is a $d$-dimensional dual hyperoval having $F$ as quotient. By a finite number of liftings, we get a $d$-dimensional dual hyperoval in $\mathrm{PG}(d(d+3) / 2,2)$ having $F$ as quotient.

Theorems 3.1, 3.2 imply:

Theorem 3.3. A characteristic d-dimensional dual hyperoval in $\operatorname{PG}(n, 2)$ is universal if and only if $n=d(d+3) / 2$.

We note that Theorem 1.2 implies:

Theorem 3.4. If $d \geqslant 6$, any characteristic d-dimensional dual hyperoval in $\mathrm{PG}(d(d+$ $3) / 2,2)$ admits quotients in $\mathrm{PG}(k, 2), 4 d \leqslant k \leqslant d(d+3) / 2$. 
In particular the above result holds for the universal dimensional dual hyperovals constructed starting from the Teirlinck SBQS's in Theorem 2.5. In our opinion, the lower bound $4 d$ should be improved for them, as it happens for the Huybrechts' family.

\section{Property (mI)}

Lemma 4.1. $A$ d-dimensional dual hyperoval satisfying property $(\mathrm{H})$ does not satisfy property $(\mathrm{mI})$ if and only if there exist six pairwise distinct elements of $V, u, v, u^{\prime}, v^{\prime}, u^{\prime \prime}$, $v^{\prime \prime}$, such that

$$
a_{u, v}+a_{u^{\prime}, v^{\prime}}=a_{u^{\prime \prime}, v^{\prime \prime}}
$$

Proof. Suppose that (34) holds. Since the elements $u, v, u^{\prime}, v^{\prime}, u^{\prime \prime}, v^{\prime \prime}$ are pairwise distinct, the intersection of $S_{u^{\prime \prime}}$ with the $2 d$-space spanned by $S_{u}$ and $S_{u^{\prime}}$ contains the point $a_{u^{\prime \prime}, v^{\prime \prime}}$. We want to prove that this point does not belong to the line $\left\langle a_{u, u^{\prime \prime}}, a_{u^{\prime}, u^{\prime \prime}}\right\rangle$. If not this line would be coplanar with the line $\left\langle a_{u, v}, a_{u^{\prime}, v^{\prime}}\right\rangle$ so that $\left\langle a_{u, v}, a_{u, u^{\prime \prime}}\right\rangle$ and $\left\langle a_{u^{\prime}, v^{\prime}}, a_{u^{\prime}, u^{\prime \prime}}\right\rangle$ would meet in the point $a_{u, u^{\prime}}$. In this case $a_{u^{\prime \prime}, v^{\prime \prime}}$ would lie on the plane determined by the pairwise meeting points of $S_{u}, S_{u^{\prime}}$ and $S_{u^{\prime \prime}}$. Property $(\mathrm{H})$ would imply $v=v^{\prime}=v^{\prime \prime}=q\left(\left\{u, u^{\prime}, u^{\prime \prime}\right\}\right)$, a contradiction. Since $a_{u^{\prime \prime}, v^{\prime \prime}}$ belongs to $\left\langle S_{u}, S_{u^{\prime}}\right\rangle-\left\langle a_{u, u^{\prime \prime}}, a_{u^{\prime}, u^{\prime \prime}}\right\rangle$ (hence $\left\langle S_{u}, S_{u^{\prime}}\right\rangle \cap S_{u^{\prime \prime}}$ is not a line), property (mI) does not hold.

If property $(\mathrm{mI})$ does not hold then there exist three pairwise distinct elements $u, u^{\prime}, u^{\prime \prime}$ in $V$ such that $\left\langle S_{u}, S_{u^{\prime}}\right\rangle \cap S_{u^{\prime \prime}}$ contains a point $a_{u^{\prime \prime}, v^{\prime \prime}}$, with $v^{\prime \prime} \notin\left\{u, u^{\prime}, u^{\prime \prime}\right.$, $\left.q\left(\left\{u, u^{\prime}, u^{\prime \prime}\right\}\right)\right\}$. Thus there exist $v$ and $v^{\prime}$ in $V$ such that

$$
a_{u, v}+a_{u^{\prime}, v^{\prime}}=a_{u^{\prime \prime}, v^{\prime \prime}} .
$$

Obviously, both $v$ and $v^{\prime}$ are different from $u$ and $u^{\prime}$. It remains to prove that $v, v^{\prime}, v^{\prime \prime}$ are pairwise distinct. If not, we may suppose $v=v^{\prime}$. In such a case, property (H) also implies $v=v^{\prime}=v^{\prime \prime}$. Thus, by property (H) and (35), we have:

$$
a_{u^{\prime \prime}, v^{\prime \prime}}=a_{u, v^{\prime \prime}}+a_{u^{\prime}, v^{\prime \prime}}=a_{q\left(\left\{u, u^{\prime}, v^{\prime \prime}\right\}\right), v^{\prime \prime}}
$$

whence $u^{\prime \prime}=q\left(\left\{u, u^{\prime}, v^{\prime \prime}\right\}\right)$, i.e., $v^{\prime \prime}=q\left(\left\{u, u^{\prime}, u^{\prime \prime}\right\}\right)$, a contradiction.

From now on, let $F$ be a characteristic $d$-dimensional dual hyperoval. As in the previous sections we work in the vector space $\mathbb{Z}_{2}^{(d+2)}$. Again we choose a basis $\mathscr{B}$ containing $z$ in $V=\mathbb{Z}_{2}^{d+1}$ and set, as in Section $2, \mathscr{G}=\mathscr{B} \cup\{0\}$.

Lemma 4.2. For any $u, u^{\prime}, u^{\prime \prime}$ in $V$, we have

$$
a_{u, u+z}+a_{u^{\prime}, u^{\prime}+z}+a_{u^{\prime \prime}, u^{\prime \prime}+z} \neq 0 .
$$

Proof. Suppose $a_{u, u+z}+a_{u^{\prime}, u^{\prime}+z}+a_{u^{\prime \prime}, u^{\prime \prime}+z}=0$. It follows from (18) that

$$
0=\sum_{w \in J(u)}\left(a_{w, 0}+a_{w, z}\right)+\sum_{w \in J\left(u^{\prime}\right)}\left(a_{w, 0}+a_{w, z}\right)+\sum_{w \in J\left(u^{\prime \prime}\right)}\left(a_{w, 0}+a_{w, z}\right) .
$$


The above equality implies $J\left(u^{\prime \prime}\right)=J(u) \triangle J\left(u^{\prime}\right)$, and then $J\left(u^{\prime \prime}\right)$ would be even, a contradiction.

Lemma 4.3. For any $u, u^{\prime}, v^{\prime}$ in $V$, we have

$$
a_{u, u+z}+a_{u^{\prime}, v^{\prime}}+a_{u^{\prime}+z, v^{\prime}+z} \neq 0 .
$$

Proof. Suppose $a_{u, u+z}+a_{u^{\prime}, v^{\prime}}+a_{u^{\prime}+z, v^{\prime}+z}=0$. It follows from (14) and (18) that

$$
0=\sum_{w \in J(u)}\left(a_{w, 0}+a_{w, z}\right)+\sum_{w \in J\left(u^{\prime}\right)}\left(a_{w, 0}+a_{w, z}\right)+\sum_{w \in J\left(v^{\prime}\right)}\left(a_{w, 0}+a_{w, z}\right) .
$$

The above equality implies $J(u)=J\left(u^{\prime}\right) \triangle J\left(v^{\prime}\right)$, and then $J(u)$ would be even, a contradiction.

We give some new notation. For every $u$ in $V$, we set $J^{*}(u)=J(u)-\{0, z\}$. For $u, v$ in $V$, we also set:

$$
\begin{aligned}
I_{u, v}=J(u) \cap J(v) & I_{u, v}^{*}=J^{*}(u) \cap J^{*}(v) \\
D_{u, v}=J(u)-J(v) & D_{u, v}^{*}=J^{*}(u)-J^{*}(v) \\
D_{v, u}=J(v)-J(u) & D_{v, u}^{*}=J^{*}(v)-J^{*}(u) \\
U_{u, v}=J(u) \cup J(v) & U_{u, v}^{*}=J^{*}(u) \cup J^{*}(v)
\end{aligned}
$$

Furthermore, for any pair of subsets $S, T$ of $\mathscr{G}$, define

$$
a_{S, T}=a_{T, S}=\sum_{\substack{s \in S \\ t \in T}} a_{S, t}
$$

Finally, define by (16) the reduced sum of the $a_{u, v}$ 's as done by (23) for the $b_{u, v}$ 's in the proof of Theorem 2.5 .

Remark 4.4. Since $J(u)$ and $J(v)$ have odd size, we deduce $\left|D_{u, v}\right| \equiv\left|D_{v, u}\right| \equiv$ $\left|I_{u, v}\right|+1(\bmod 2)$. If $w \in I_{u, v}^{*}$, then the number $y_{w}$ of elements $a_{w, x}$ in the reduced sum of $a_{u, v}$ equals either $\left|D_{u, v}\right|+\left|D_{v, u}\right|$ or $\left|D_{u, v}\right|+\left|D_{v, u}\right| \pm 2$ (taking into account the possible term $\left.a_{w, 0}+a_{w, z}\right)$ so that $y_{w}$ is even. If $w \in D_{u, v}^{*}$, then the number $y_{w}$ of elements $a_{w, x}$ in the reduced sum of $a_{u, v}$ equals either $|J(v)|$ or $|J(v)| \pm 2$ so that $y_{w}$ is odd. Analogously it happens for the elements $w$ in $D_{v, u}^{*}$.

Proposition 4.5. Let $a_{u, v}+a_{u^{\prime}, v^{\prime}}+a_{u^{\prime \prime}, v^{\prime \prime}}=0$. Every $w \in\left(U_{u, v} \cup U_{u^{\prime}, v^{\prime}} \cup U_{u^{\prime \prime}, v^{\prime \prime}}\right)-$ $\{0, z\}$ belongs to at least two sets among $U_{u, v}, U_{u^{\prime}, v^{\prime}}, U_{u^{\prime \prime}, v^{\prime \prime}}$ and belongs to 0 or 2 sets among $D_{u, v}, D_{v, u}, D_{u^{\prime}, v^{\prime}}, D_{v^{\prime}, u^{\prime}}, D_{u^{\prime \prime}, v^{\prime \prime}}, D_{v^{\prime \prime}, u^{\prime \prime}}$.

Proof. The equality in the hypothesis implies that the number of distinct elements 
$a_{w, x}$ in the sum of the three reduced sums of $a_{u, v}, a_{u^{\prime}, v^{\prime}}, a_{u^{\prime \prime}, v^{\prime \prime}}$ is even. The statement follows from Remark 4.4 .

Theorem 4.6. Every characteristic d-dimensional dual hyperoval in $\mathrm{PG}(d(d+3) / 2,2)$ satisfies property $(\mathrm{mI})$.

Proof. According to Lemma 4.1, it is enough to prove that equality (34) does not hold for any choice of pairwise distinct elements $u, v, u^{\prime}, v^{\prime}, u^{\prime \prime}, v^{\prime \prime}$ in $V$. Set

$$
\beta_{u, v}=\sum_{w \in J(v)} x_{w, u}\left(a_{w, 0}+a_{w, z}\right)+x_{u, v} \sum_{w \in J(u)}\left(a_{w, 0}+a_{w, z}\right) .
$$

Using (16) and (39), equality (34) becomes

$$
\begin{aligned}
& 0=a_{I_{u, v}, D_{u, v}}+a_{I_{u, v}, D_{c, u}}+a_{D_{u, v}, D_{v, u}}+\beta_{u, v} \\
& +a_{I_{u^{\prime}, v^{\prime}}, D_{u^{\prime}, r^{\prime}}}+a_{I_{u^{\prime}, r^{\prime}}, D_{r^{\prime}, u^{\prime}}}+a_{D_{u^{\prime}, \mathrm{r}^{\prime}}, D_{r^{\prime}, u^{\prime}}}+\beta_{u^{\prime}, v^{\prime}} \\
& +a_{I_{u^{\prime \prime}, v^{\prime \prime}}, D_{u^{\prime \prime}, v^{\prime \prime}}}+a_{I_{u^{\prime \prime}, v^{\prime \prime}}, D_{v^{\prime \prime}, u^{\prime \prime}}}+a_{D_{u^{\prime \prime}, v^{\prime \prime}}, D_{v^{\prime \prime}, u^{\prime \prime}}}+\beta_{u^{\prime \prime}, v^{\prime \prime}} .
\end{aligned}
$$

Since the points $a_{x, y}$ with $x, y$ distinct elements of $\mathscr{G}$ (and in particular of $\mathscr{G}-\{0, z\}$ ) are independent in $\operatorname{PG}(d(d+3) / 2,2)$, equality (40) implies

$$
\begin{aligned}
0= & a_{I_{u, v}^{*}, D_{u, v}^{*}}+a_{I_{u, v}^{*}, D_{v^{*}, u}^{*}}+a_{D_{u, v}^{*}, D_{v, u}^{*}} \\
& +a_{I_{u^{\prime}, v^{\prime}}^{*}, D_{u^{\prime}, x^{\prime}}^{*}}+a_{I_{u^{\prime}, v^{\prime}}^{*}, D_{r^{\prime}, u^{\prime}}^{*}}+a_{D_{u^{\prime}, v^{\prime}}^{*}, D_{v^{\prime}, u^{\prime}}^{*}} \\
& +a_{I_{u^{\prime \prime}, v^{\prime \prime}}^{*}, D_{u^{\prime \prime}, v^{\prime \prime}}^{*}}+a_{I_{u^{\prime \prime}, v^{\prime \prime}}^{*}, D_{v^{\prime \prime}, u^{\prime \prime}}^{*}}+a_{D_{u^{\prime \prime}, v^{\prime \prime}}^{*}, D_{v^{\prime \prime}, u^{\prime \prime}}^{*}}
\end{aligned}
$$

With

$$
\begin{array}{llll}
A=I_{u, v}-U_{u^{\prime}, v^{\prime}}, & A^{\prime}=I_{u^{\prime}, v^{\prime}}-U_{u, v}, & B=I_{u, v} \cap I_{u^{\prime}, v^{\prime}}, & \\
E=I_{u, v} \cap D_{u^{\prime}, v^{\prime}}, & F=I_{u, v} \cap D_{v^{\prime}, u^{\prime}}, & E^{\prime}=I_{u^{\prime}, v^{\prime}} \cap D_{u, v}, & F^{\prime}=I_{u^{\prime}, v^{\prime}} \cap D_{v, u}, \\
H=D_{u, v}-U_{u^{\prime}, v^{\prime}}, & K=D_{v, u}-U_{u^{\prime}, v^{\prime}}, & H^{\prime}=D_{u^{\prime}, v^{\prime}}-U_{u, v}, & K^{\prime}=D_{v^{\prime}, u^{\prime}}-U_{u, v}, \\
P=D_{u, v} \cap D_{u^{\prime}, v^{\prime}}, & Q=D_{v, u} \cap D_{v^{\prime}, u^{\prime}}, & X=D_{u, v} \cap D_{v^{\prime}, u^{\prime}}, & Y=D_{v, u} \cap D_{u^{\prime}, v^{\prime}},
\end{array}
$$

we have

$$
\begin{aligned}
& a_{I_{u, v}^{*}, D_{u, v}^{*}}+a_{I_{u, v}^{*}, D_{v, u}^{*}}+a_{D_{u, v}^{*}, D_{i, u}^{*}}=a_{A, E^{\prime}}+a_{A, H}+a_{A, P}+a_{A, X}+a_{A, F^{\prime}}+a_{A, K} \\
& +a_{A, Q}+a_{A, Y}+a_{B, E^{\prime}}+a_{B, H}+a_{B, P}+a_{B, X}+a_{B, F^{\prime}}+a_{B, K}+a_{B, Q}+a_{B, Y}+a_{E, E^{\prime}} \\
& +a_{E, H}+a_{E, P}+a_{E, X}+a_{E, F^{\prime}}+a_{E, K}+a_{E, Q}+a_{E, Y}+a_{F, E^{\prime}}+a_{F, H}+a_{F, P}+a_{F, X} \\
& +a_{F, F^{\prime}}+a_{F, K}+a_{F, Q}+a_{F, Y}+a_{E^{\prime}, F^{\prime}}+a_{E^{\prime}, K}+a_{E^{\prime}, Q}+a_{E^{\prime}, Y}+a_{H, F^{\prime}}+a_{H, K} \\
& +a_{H, Q}+a_{H, Y}+a_{P, F^{\prime}}+a_{P, K}+a_{P, Q}+a_{P, Y}+a_{X, F^{\prime}}+a_{X, K}+a_{X, Q}+a_{X, Y}
\end{aligned}
$$


$a_{I_{u^{\prime}, v^{\prime}}^{*}, D_{u^{\prime}, v^{\prime}}^{*}}+a_{I_{u^{\prime}, v^{\prime}}^{*}, D_{v^{\prime}, u^{\prime}}^{*}}+a_{D_{u^{\prime}, v^{\prime}}^{*}, D_{r^{\prime}, u^{\prime}}^{*}}=a_{A^{\prime}, E}+a_{A^{\prime}, H^{\prime}}+a_{A^{\prime}, P}+a_{A^{\prime}, Y}+a_{A^{\prime}, F}$

$+a_{A^{\prime}, K^{\prime}}+a_{A^{\prime}, Q}+a_{A^{\prime}, X}+a_{B, E}+a_{B, H^{\prime}}+a_{B, P}+a_{B, Y}+a_{B, F}+a_{B, K^{\prime}}+a_{B, Q}+a_{B, X}$

$+a_{E^{\prime}, E}+a_{E^{\prime}, H^{\prime}}+a_{E^{\prime}, P}+a_{E^{\prime}, Y}+a_{E^{\prime}, F}+a_{E^{\prime}, K^{\prime}}+a_{E^{\prime}, Q}+a_{E^{\prime}, X}+a_{F^{\prime}, E}+a_{F^{\prime}, H^{\prime}}$

$+a_{F^{\prime}, P}+a_{F^{\prime}, Y}+a_{F^{\prime}, F}+a_{F^{\prime}, K^{\prime}}+a_{F^{\prime}, Q}+a_{F^{\prime}, X}+a_{E, F}+a_{E, K^{\prime}}+a_{E, Q}+a_{E, X}+a_{H^{\prime}, F}$ $+a_{H^{\prime}, K^{\prime}}+a_{H^{\prime}, Q}+a_{H^{\prime}, X}+a_{P, F}+a_{P, K^{\prime}}+a_{P, Q}+a_{P, X}+a_{Y, F}+a_{Y, K^{\prime}}+a_{Y, Q}+a_{Y, X}$.

It follows from (41) that

$$
\begin{aligned}
& a_{I_{u^{\prime \prime}, \mathrm{r}^{\prime \prime}}, D_{u^{\prime \prime}, \mathrm{r}^{\prime \prime}}^{*}}+a_{I_{u^{\prime \prime}, \mathrm{v}^{\prime \prime}}^{*}, D_{\mathbf{r}^{\prime \prime}, \mathrm{u}^{\prime \prime}}^{*}}+a_{D_{u^{\prime \prime}, \mathrm{v}^{\prime \prime}}^{*}, D_{\mathbf{v}^{\prime \prime}, u^{\prime \prime}}^{*}}=a_{A, E^{\prime}}+a_{A, H}+a_{A, P} \\
& +a_{A, X}+a_{A, F^{\prime}}+a_{A, K}+a_{A, Q}+a_{A, Y}+a_{A^{\prime}, E}+a_{A^{\prime}, H^{\prime}}+a_{A^{\prime}, P}+a_{A^{\prime}, Y} \\
& +a_{A^{\prime}, F}+a_{A^{\prime}, K^{\prime}}+a_{A^{\prime}, Q}+a_{A^{\prime}, X}+a_{B, E^{\prime}}+a_{B, H}+a_{B, F^{\prime}}+a_{B, K}+a_{B, E} \\
& +a_{B, H^{\prime}}+a_{B, F}+a_{B, K^{\prime}}+a_{E, H}+a_{E, P}+a_{E, K}+a_{E, Y}+a_{E, F}+a_{E, K^{\prime}} \\
& +a_{F, H}+a_{F, X}+a_{F, K}+a_{F, Q}+a_{E^{\prime}, F^{\prime}}+a_{E^{\prime}, K}+a_{E^{\prime}, H^{\prime}}+a_{E^{\prime}, P}+a_{E^{\prime}, K^{\prime}} \\
& +a_{E^{\prime}, X}+a_{F^{\prime}, H^{\prime}}+a_{F^{\prime}, Y}+a_{F^{\prime}, K^{\prime}}+a_{F^{\prime}, Q}+a_{H, F^{\prime}}+a_{H, K}+a_{H, Q}+a_{H, Y} \\
& +a_{H^{\prime}, F}+a_{H^{\prime}, K^{\prime}}+a_{H^{\prime}, Q}+a_{H^{\prime}, X}+a_{P, K}+a_{P, Y}+a_{P, K^{\prime}}+a_{P, X}+a_{X, K} \\
& +a_{X, Q}+a_{Y, K^{\prime}}+a_{Y, Q} .
\end{aligned}
$$

By Proposition 4.5, $I_{u^{\prime \prime}, v^{\prime \prime}}^{*}$ contains $A, A^{\prime}$ and a (possibly empty) set $T$ which is the union of some sets among $B, P, Q, X, Y$, while $D_{u^{\prime \prime}, v^{\prime \prime}}^{*} \cup D_{v^{\prime \prime}, u^{\prime \prime}}^{*}$ contains $E, F, E^{\prime}, F^{\prime}$, $H, K, H^{\prime}, K^{\prime}$.

The presence of any non-empty set among the last ones either in $D_{u^{\prime \prime}, v^{\prime \prime}}^{*}$ or in $D_{v^{\prime \prime}, u^{\prime \prime}}^{*}$ implies some conditions for the position of some other ones. We only explicitely declare the conditions related to the presence in $D_{u^{\prime \prime}, v^{\prime \prime}}^{*}$ :

$$
\begin{array}{lll}
E \neq \varnothing, & E \subseteq D_{u^{\prime \prime}, v^{\prime \prime}}^{*} \Rightarrow D_{v^{\prime \prime}, u^{\prime \prime}}^{*}=F \cup H \cup K \cup K^{\prime}, & T=B \cup P \cup Y \\
F \neq \varnothing, & F \subseteq D_{u^{\prime \prime}, v^{\prime \prime}}^{*} \Rightarrow D_{v^{\prime \prime}, u^{\prime \prime}}^{*}=E \cup H \cup K \cup H^{\prime}, & T=B \cup Q \cup X \\
E^{\prime} \neq \varnothing, & E^{\prime} \subseteq D_{u^{\prime \prime}, v^{\prime \prime}}^{*} \Rightarrow D_{v^{\prime \prime}, u^{\prime \prime}}^{*}=F^{\prime} \cup H^{\prime} \cup K \cup K^{\prime}, & T=B \cup P \cup X \\
F^{\prime} \neq \varnothing, & F^{\prime} \subseteq D_{u^{\prime \prime}, v^{\prime \prime}}^{*} \Rightarrow D_{v^{\prime \prime}, u^{\prime \prime}}^{*}=E^{\prime} \cup H \cup H^{\prime} \cup K^{\prime}, & T=B \cup Q \cup Y \\
H \neq \varnothing, & H \subseteq D_{u^{\prime \prime}, v^{\prime \prime}}^{*} \Rightarrow D_{v^{\prime \prime}, u^{\prime \prime}}^{*}=E \cup F \cup F^{\prime} \cup K, & T=B \cup Q \cup Y \\
K \neq \varnothing, & K \subseteq D_{u^{\prime \prime}, v^{\prime \prime}}^{*} \Rightarrow D_{v^{\prime \prime}, u^{\prime \prime}}^{*}=E \cup F \cup E^{\prime} \cup H, & T=B \cup P \cup X \\
H^{\prime} \neq \varnothing, & H^{\prime} \subseteq D_{u^{\prime \prime}, v^{\prime \prime}}^{*} \Rightarrow D_{v^{\prime \prime}, u^{\prime \prime}}^{*}=F \cup E^{\prime} \cup F^{\prime} \cup K^{\prime}, & T=B \cup Q \cup X \\
K^{\prime} \neq \varnothing, & K^{\prime} \subseteq D_{u^{\prime \prime}, v^{\prime \prime}}^{*} \Rightarrow D_{v^{\prime \prime}, u^{\prime \prime}}^{*}=E \cup E^{\prime} \cup F^{\prime} \cup H^{\prime}, & T=B \cup P \cup Y
\end{array}
$$

The presence of $a_{P, Y}, a_{P, X}, a_{X, Q}, a_{Y, Q}$ in equality (42) also implies

$$
P=Q=\varnothing \quad \text { or } \quad X=Y=\varnothing
$$


while the presence of $a_{A, P}, a_{A, X}, a_{A, Q}, a_{A, Y}, a_{A^{\prime}, P}, a_{A^{\prime}, Y}, a_{A^{\prime}, Q}, a_{A^{\prime}, X}$ in equality (42) implies

$$
A \cup A^{\prime} \neq \varnothing \Rightarrow P=Q=X=Y=\varnothing .
$$

The absence of $a_{A, H^{\prime}}, a_{A, K^{\prime}}, a_{A, E}, a_{A, F}$ in (42) implies

$$
A \neq \varnothing \Rightarrow H^{\prime}=K^{\prime}=E=F=\varnothing
$$

while the absence of $a_{A^{\prime}, H}, a_{A^{\prime}, K}, a_{A^{\prime}, E^{\prime}}, a_{A^{\prime}, F^{\prime}}$, implies

$$
A^{\prime} \neq \varnothing \Rightarrow H=K=E^{\prime}=F^{\prime}=\varnothing .
$$

If $A \neq \varnothing$ and $A^{\prime} \neq \varnothing$, it follows from (45), (46) and (47)

$$
\begin{aligned}
& I_{u, v}=A \cup B, \quad D_{u, v}=\varnothing, \quad D_{v, u}=\varnothing ; \\
& I_{u^{\prime}, v^{\prime}}=A^{\prime} \cup B, \quad D_{u^{\prime}, v^{\prime}}=\varnothing, \quad D_{v^{\prime}, u^{\prime}}=\varnothing \text {; } \\
& I_{u^{\prime \prime}, v^{\prime \prime}}=A \cup A^{\prime} \cup B, \quad D_{u^{\prime \prime}, v^{\prime \prime}}=\varnothing, \quad D_{v^{\prime \prime}, u^{\prime \prime}}=\varnothing \text {. }
\end{aligned}
$$

Thus we have $v=u+z, v^{\prime}=u^{\prime}+z, v^{\prime \prime}=u^{\prime \prime}+z$ and equality (34) does not hold by Lemma 4.2.

If $A \neq \varnothing$ and $A^{\prime}=\varnothing$, by (45), (46) we have, up to exchange between $D_{u^{\prime \prime}, v^{\prime \prime}}$ and $D_{v^{\prime \prime}, u^{\prime \prime}}$

$$
\begin{aligned}
I_{u, v} & =A \cup B, & D_{u, v} & =E^{\prime} \cup H, & D_{v, u} & =F^{\prime} \cup K ; \\
I_{u^{\prime}, v^{\prime}} & =B \cup E^{\prime} \cup F^{\prime}, & D_{u^{\prime}, v^{\prime}} & =\varnothing, & D_{v^{\prime}, u^{\prime}} & =\varnothing ; \\
I_{u^{\prime \prime}, v^{\prime \prime}} & =A \cup B, & D_{u^{\prime \prime}, v^{\prime \prime}} & =E^{\prime} \cup H, & D_{v^{\prime \prime}, u^{\prime \prime}} & =F^{\prime} \cup K .
\end{aligned}
$$

Thus $v^{\prime}=u^{\prime}+z, u^{\prime \prime}=u+z$ and $v^{\prime \prime}=v+z$ and equality (34) does not hold by Lemma 4.3.

Analogously we also exclude the case $A=\varnothing, A^{\prime} \neq \varnothing$.

The case $A=A^{\prime}=\varnothing$ can be discussed in a similar way, distinguishing some subcases according to the non-empty sets among $E, E^{\prime}, F, F^{\prime}, H, H^{\prime}, K, K^{\prime}$. In every case one gets a contradiction, using Lemmas $4.2,4.3$ or falling in a coincidence between two elements among $u, u^{\prime}, v, v^{\prime}, u^{\prime \prime}, v^{\prime \prime}$.

Theorem 4.6 includes Huybrechts' family (for which property $(\mathrm{mI})$ is already proved in [6]) and the dimensional dual hyperovals constructed in Theorem 2.5.

\section{Open problems}

We have proved that $d(d+3) / 2$ is the upper bound for the dimension of a projective space containing a characteristic $d$-dimensional dual hyperoval. Is this true for any $d$ dimensional dual hyperoval satisfying property $(\mathrm{H})$, or better, for any $d$-dimensional dual hyperoval?

Boolean and Teirlinck systems are associated with some dimensional dual hyper- 
ovals. Are there other (possibly characteristic) SBQS's related to dimensional dual hyperovals satisfying property $(\mathrm{H})$ ? In view of Remark 2.4 , we conjecture that the answer is negative. In other words, the link between SBQSs and dimensional dual hyperovals does not appear so strong as we suspected at the beginning of our research on this subject.

As noticed by Huybrechts in [6], Huybrechts' family gives rise to semi-biplanes (see Section 1.3) that are quotients of upper 3-truncated Coxeter complexes. Are the semibiplanes defined by the dimensional dual hyperovals of Theorem 2.5 completely new?

Acknowledgement. The authors wish to thank S. Yoshiara for having pointed out useful information about the family a) in the table of Section 1.2.

\section{References}

[1] M. Buratti, A. Del Fra, A lower bound on the number of semi-Boolean quadruple systems. To appear in J. Combin. Des.

[2] J. H. Conway, R. T. Curtis, S. P. Norton, R. A. Parker, R. A. Wilson, Atlas of finite groups. Oxford Univ. Press 1985. MR 88g:20025 Zbl 0568.20001

[3] B. N. Cooperstein, J. A. Thas, On generalized $k$-arcs in PG(2n, q). Ann. Comb. 5 (2001), 141-152. MR 2003c:51005 Zbl 01756461

[4] A. Del Fra, On $d$-dimensional dual hyperovals. Geom. Dedicata 79 (2000), 157-178. MR 2001f:51014 Zbl 0948.51008

[5] J. W. P. Hirschfeld, Projective geometries over finite fields. Oxford Univ. Press 1979. MR 81h:51007 Zbl 0418.51002

[6] C. Huybrechts, Dimensional dual hyperovals in projective spaces and c.AG*-geometries. Discrete Math. 255 (2002), 193-223. MR 1927795 Zbl 01838892

[7] C. Huybrechts, A. Pasini, Flag-transitive extensions of dual affine spaces. Beiträge Algebra Geom. 40 (1999), 503-531. MR 2001f:51019 Zbl 0957.51004

[8] L. Teirlinck, Combinatorial properties of planar spaces and embeddability. J. Combin. Theory Ser. A 43 (1986), 291-302. MR 88g:51006 Zbl 0605.51009

[9] J. A. Thas, H. Van Maldeghem, Characterization of the finite quadric and Hermitian Veroneseans over finite fields. To appear in J. Geom.

[10] S. Yoshiara, A family of $d$-dimensional dual hyperovals in $\mathrm{PG}(2 d+1,2)$. European $J$. Combin. 20 (1999), 589-603. MR 2001b:51016 Zbl 0937.51009

[11] S. Yoshiara, On a family of planes of a polar space. European J. Combin. 22 (2001), 107-118. MR 2001m:51011 Zbl 0971.51003

[12] S. Yoshiara, Ambient spaces of dimensional dual arcs. To appear in J. Algebraic Combin.

Received 22 January, 2003; revised 13 March, 2003

M. Buratti, Dipartimento di Matematica e Informatica, Università di Perugia, Via Vanvitelli 1, I-06123 Perugia, Italy.

Email: buratti@mat.uniroma1.it

A. Del Fra, Dipartimento di Metodi e Modelli Matematici per le Scienze Applicate, Università di Roma "La Sapienza", Via Scarpa 16, I-00161 Roma, Italy.

Email: alberto.delfra@uniromal.it 